\title{
A self-assembled luminescent host that selectively senses ATP in water.
}

\begin{abstract}
Metal-ion-directed self-assembly has been used to construct kinetically inert, water-soluble heterometallic $\mathrm{Ru} 2 \mathrm{Re} 2$ hosts that are potential sensors for bioanions. A previously reported metallomacrocycle and a new derivative synthesised by this approach are found to be general sensors for bioanions in water, showing an "off-on" luminescent change that is selective for nucleotides over uncharged nucleobases. Through a change in the ancillary ligands coordinated to the ruthenium centres of the host, an "off-on" sensor has been produced. Whilst this host only shows a modest enhancement in binding affinities for nucleotides relative to the other two host systems, its sensing response is much more specific. Although a distinctive "off-on" luminescence response is observed for the addition of adenosine triphosphosphate (ATP), related structures such as adenine and guanosine triphosphate (GTP) do not induce any emission change in the host. Detailed and demanding DFT studies on the ATP- and GTP-bound host-guest complexes reveal subtle differences in their geometries that modulate the stacking interactions between the nucleotide guests and the ancillary ligands of the host. It is suggested that this change in stacking geometries affects solvent accessibility to the binding pocket of the host and thus leads to observed difference in the host luminescence response to the guests.
\end{abstract}

Keyword: Density functional calculations; Luminescence; Nucleotides; Self-assembly; Sensors. 